\title{
Feasibility of digitally stained multimodal confocal mosaics to simulate histopathology
}

\author{
Daniel S. Gareau \\ Sloan Kettering Cancer Center \\ Dermatology Service \\ 160 East 53rd Street \\ New York, New York 10022 \\ and \\ Oregon Health \& Science University \\ Departments of Dermatology \\ and Biomedical Engineering \\ 3303 S.W. Bond Avenue \\ Portland, Oregon 97239
}

\begin{abstract}
Fluorescence confocal mosaicing microscopy of tissue biopsies stained with acridine orange has been shown to accurately identify tumors and with an overall sensitivity of $96.6 \%$ and specificity of $89.2 \%$. However, fluorescence shows only nuclear detail similar to hematoxylin in histopathology and does not show collagen or cytoplasm, which may provide necessary negative contrast information similar to eosin used in histopathology. Reflectance mode contrast is sensitive to collagen and cytoplasm without staining. To further improve sensitivity and specificity, digitally stained confocal mosaics combine confocal fluorescence and reflectance images in a multimodal pseudo-color image to mimic the appearance of histopathology with hematoxylin and eosin and facilitate the introduction of confocal microscopy into the clinical realm. ๑ 2009 Society of Photo-Optical Instrumentation Engineers. [DOI: 10.1117/1.3149853]
\end{abstract}

Keywords: scanning microscopy; digital processing; color; fluorescence; reflectance; detection.

Paper 09041LR received Feb. 11, 2009; revised manuscript received Apr. 16, 2009; accepted for publication Apr. 21, 2009; published online Jun. 30, 2009.

\section{Introduction}

Histopathology with hematoxylin and eosin (H\&E) staining is a widely used diagnostic to guide surgical removal of cancer although it requires highly skilled technicians and is expensive and time consuming, requiring a half-hour (frozen sections) to days (permanent sections). By combining optical sectioning of whole-mount tissue excisions with large fields of view at high resolution, confocal mosaics may provide a rapid alternative and enable bedside pathology. This approach has been proven for basal cell carcinoma $(\mathrm{BCC})^{1-4}$ and is likely applicable to other tumor types.

In reflectance mode, ${ }^{1,2}$ the acetowhitened bright contrast of nuclei is modest because of the background reflectance of keratin and collagen, enabling detection of only large nodulartype tumors. Because of their diminutive size within brightly reflective collagen, tiny infiltrative tumor strands were not reliably detected in reflectance mode alone. ${ }^{2}$ Alternately, in fluorescence mode using the nuclear stain acridine orange to contrast the heavily nucleated BCC tumors, the screening capacity of the technique includes tiny infiltrative-type tumors as well as the nodular type. ${ }^{3}$ Nuclei can be brightly stained in fluorescence mode with acridine orange, whereas the cytoplasm and collagen appear dark. This selective labeling of the nuclei parallels the use of hematoxylin in conventional histopathology. The confocal microscope used in this study has been previously described. ${ }^{3}$ It provides up to 100 -fold nuclear fluorescence contrast with a field of view of $\sim 2 \mathrm{~cm}$ and submicron resolution. In a clinical study that blindly evaluated the fluorescence confocal diagnosis against the gold standard

Address all correspondence to Daniel Gareau, Oregon Health \& Science University, Mail code: $\mathrm{CH} 13 \mathrm{~B}-3303$ SW Bond Avenue, Portland, OR 97239 United States of America; Tel: 503708 7078; Fax: 212308 0854; E-mail: dan@dangareau.net of pathology, the overall sensitivity and specificity were 96.6 and $89.2 \%$, respectively. ${ }^{4}$ These results show that the fluorescence mode is highly sensitive and specific, but to match the cure rate of Mohs surgery, which is up to $99 \%,{ }^{5}$ additional modality may be required.

The goal of this work is to combine fluorescence and reflectance modes. The reflectance mode is used not to label nuclei as in Refs. 1 and 2, but to label cytoplasm and the dermal collagen. Without the use of acetic acid to brighten nuclei, the heavily nucleated tumors appear dark while the dermal collagen and cytoplasm are labeled with endogenous bright contrast. Collagen fibers and organelles in cytoplasm scatter visible light while uncondensed chromatin fibers in nuclei do not. The contrast in reflectance mode therefore parallels the use of eosin, which also labels cytoplasm and dermal collagen. Fluorescent nuclear contrast and reflectance countercontrast can be combined using color to mimic H\&E.

Previous studies ${ }^{4}$ included two clinician readers accustomed to gray-scale fluorescence mosaics. The sensitivity of the Mohs fellow who had two months of confocal experience was $94.4 \%$, whereas the seasoned Mohs surgeon with 10 years of confocal experience was $98.9 \%$ sensitive. To gain widespread acceptance, confocal microscopy must be at least $99 \%$ sensitive and easily read by any pathologist who is accustomed to H\&E staining, such as the Mohs fellow. Toward this end, multimodal, color-coded digitally stained confocal mosaics (DSCMs) are presented. The fluorescence mode gray-scale mosaic can be assigned the purple color associated with nuclei and heavily nucleated tumors. The reflectance mode gray-scale mosaic can be assigned the pink color associated with cytoplasm and dermal collagen. Confocal fluores-

1083-3668/2009/14(3)/034050/5/\$25.00 @ 2009 SPIE 
cence and reflectance modes can emulate the contrast of $\mathrm{H} \& \mathrm{E}$, respectively.

\section{Mosaic Construction}

The samples consisted of the fresh frozen-section tissue blocks that are routinely discarded: whole tissue that approximates directly excised specimens. This sample type was chosen so that this study would not impact patient care, although ideally, samples would go directly from the patient to the confocal microscope, bypassing histopathology entirely. Samples were prepared for imaging as previously described. ${ }^{3}$ Briefly, the samples were stained in 1-mM acridine orange solution for $20 \mathrm{~s}$, dipped in a saline rinse, and compressed against a glass slide for confocal imaging. A set of images was acquired in a step-and-capture routine that covered the entire surface area (margin) of the whole-mount excision. To assure complete coverage, $10 \%$ image overlap (in both the $x$ and $y$ directions) was included. Adjacent images were stitched together seamlessly by determining the proper offset between the frame centers to match the sample features and averaging the frames in the overlapped region. The feature-matching algorithm (www.dangareau.net/TaylorStitch/index.html) written in Matlab (MathWorks, Natick, Massachusetts) included an automated iterative guessing routine to determine the proper offset position. Starting with an initial estimate of the offset between adjacent frames, the routine computed the mean pixel difference in the overlapping region. On subsequent iterations, the mean pixel difference (feature-matching error) was minimized, leading to the correct offset.

\section{Digital Staining}

The gray-scale fluorescence-only and reflectance-only mosaics were digitally stained with color and combined. Because pathologists and Mohs surgeons readily interpret H\&E staining, the purple and pink colors of these two stains were adopted to maximize association with cells and noncellular structures, respectively. To acquire the respective red, green, and blue levels required to produce these colors, a single digital image was captured of a conventionally stained (frozensection) specimen. Sampling the histological image over areas of hematoxylin staining and then eosin staining yielded the brightness of the red, green, and blue components for each stain. The experimentally determined colors for the stains were $\mathrm{H}=\left[\begin{array}{lll}0.30 & 0.20 & 1\end{array}\right]$ and $\mathrm{E}=\left[\begin{array}{lll}1 & 0.55 & 0.88\end{array}\right]$, respectively, where $[$ red $(k=1)$ green $(k=2)$ blue $(k=3)]$. The H\&E pseudo-colored confocal mosaic was computed as follows:

$$
\operatorname{DSCM}_{x, y, k}=1-F_{x, y}\left(1-H_{k}\right)-R_{x, y}\left(1-E_{k}\right) .
$$

The three-color codes $H$ and $E$ were weighted by the fluorescence $F$ (nuclear) and reflectance $R$ (collagen), where $F$ and $R$ were single values for each pixel, normalized to make the brightest pixel unity. Application of Eq. (1) to each pixel of the image yields the color image DSCM, in which nuclei appear purple and collagen and cytoplasm appear pink. Equation (1) effectively converts luminous information (reflectance and fluorescence confocal light measurements) to pigment or absorbance-based information by inverting the brightness contrast. In the absence of a fluorescent or reflective signal $(R=F=0)$, the DSCM is unity, faithfully reproducing the white areas outside the specimen in conventional H\&E histopathology. When $R$ and $F$ are nonzero, their magnitude determines the suppression of brightness in the DSCM. If the density of contrast agent (i.e., fluorescent molecules or scattering particles) for a particular sample volume element (voxel) is high, the $F$ or $R$ confocal mosaic is bright on a black background and the DSCM is dark with digitally stained color on a white background. The pseudo-colored mosaic DSCM has the appropriate dimensions for export as an image file such as bitmap, tif or jpeg.

\section{Clinical Feasibility Study}

A team of two Mohs surgeons and two pathologists blindly evaluated the DSCMs for mosaic quality and tumor presence. Ten cases were chosen from 25 to represent the challenging tumor subtypes as well as normal tissue: six micronodular, two infiltrative, and two normal. Fifteen mosaics were rejected because they either contained large nodular tumors that were obvious or the mosaic construction was less than ideal, leaving grid lines that distracted the pathologists and surgeons. DSCM quality was assessed for uniform coloring and absence of saturation and grid lines at the mosaic stitching seams. DSCMs were evaluated by visual comparison of the color tone against correlating $\mathrm{H} \& \mathrm{E}$ histopathology prepared during surgery. For each case studied, the DSCM was classified as normal, micronodular BCC, or infiltrative BCC, then compared to the correlating frozen sections previously used for intraoperative margin assessment during the Mohs procedure. This review took place about three weeks after the surgery to create a "blinded" condition as in Ref. 4.

\section{Results}

Confocal imaging on the entire margin of excised tissue from the cryostat enabled physical correlation to the histology. DSCM color correlation was achieved by digital staining of the multimodal confocal mosaics. The bright cellular features in the gray-scale fluorescence mosaics [Fig. 1(b)] due to their stained nuclei were identified as purple in the DSCM [Fig. 1(c)]. Dermal collagen, cytoplasm, and keratin in the stratum corneum appeared bright in the gray-scale reflectance mosaics [Fig. 1(a)] and pink in the DSCM [Fig. 1(c)]. For each of the 10 cases (including two negative samples), the DSCM lead to the correct identification of normal and malignant morphology compared to the gold-standard histology. Well-ordered nuclei characterized normal cellular tissue components, such as the healthy epidermis. Tumor nuclei showed crowding, disarray, and irregular sizes. Figure 1(e), 1(f), and 1(j) shows $30 \times$ views of the micronodular BCC [Fig. 1(e)] and superficial epidermis [Fig. 1(f)] indicated in Fig. 1(c). Figure $1(\mathrm{~g})-1(\mathrm{j})-1(\mathrm{~J})$ shows an example of infiltrative BCC with the presence of heavy inflammation and dermal collagen, representing the hardest tumor type to detect. 

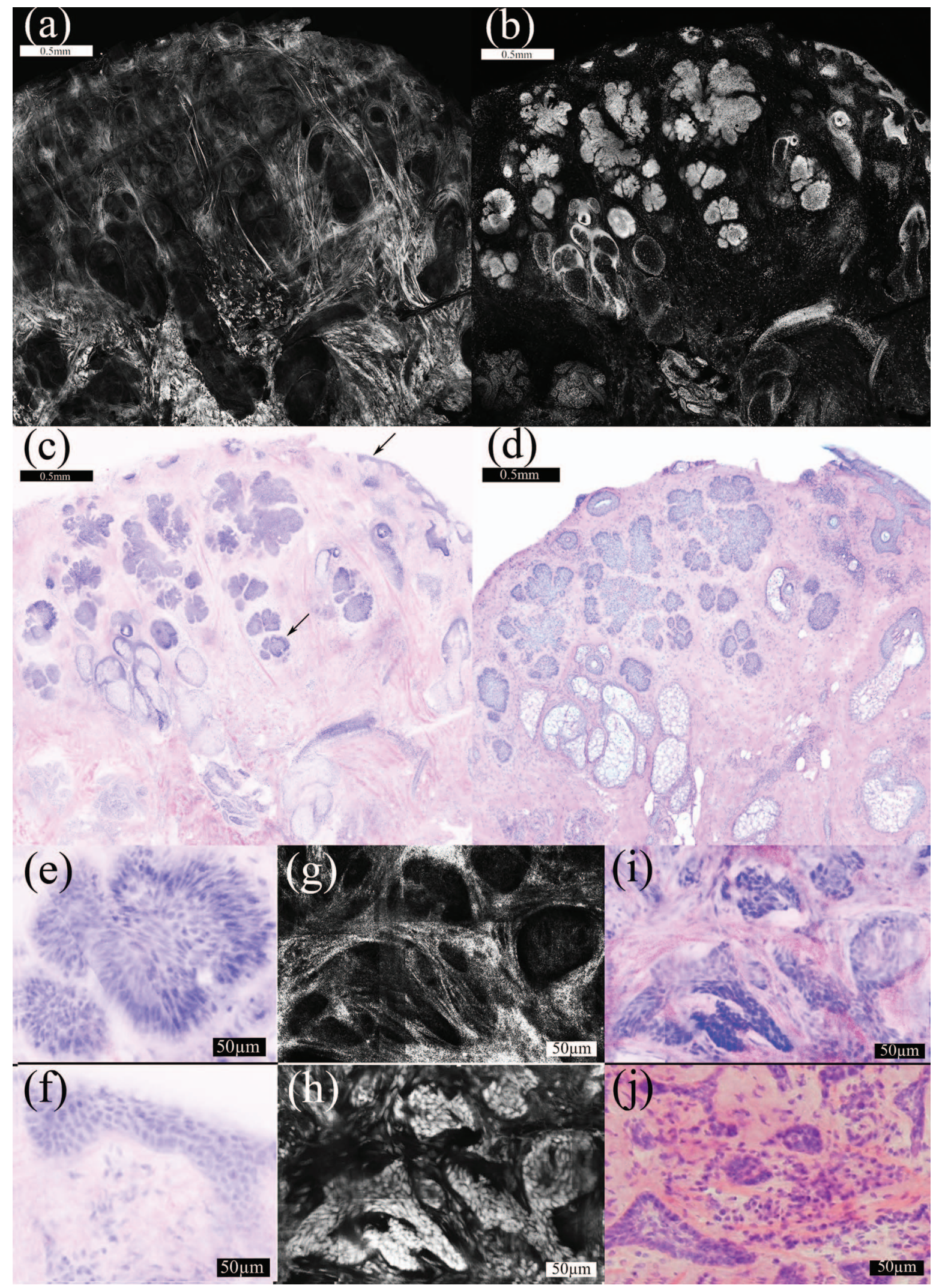

Fig. 1 (a-d) $2 \times$ magnification. The reflectance mosaic (a) is pink, and the fluorescence mosaic (b) is purple in the DSCM image (c). The correlating H\&E histopathology $(d)$ is also shown at $2 \times$ magnification. The arrows (c) indicate a micronodular focus in the deeper dermis and the superficial epidermis. (e-j) 30× magnification. A micronodular tumor focus in the dermis (e) and normal superficial epidermis (f) are magnified from (c). In a separate sample containing infiltrative collagen is bright in the deeper dermis in reflectance mode $(\mathrm{g})$. The correlating fluorescence image (h) shows bright nuclei. The DSCM image (i) is shown with the correlating histopathology (j). 


\section{Discussion}

For BCC, two or more modes of contrast (i.e., multimodal contrast) are likely necessary: a "positive" mode for detecting the presence of nucleated tumors and a "negative" mode for detecting the absence of tumor (i.e., presence of normal tissue, such as collagen or cytokeratin). The work presented here implements multimodal fluorescence/reflectance confocal imaging that mimics H\&E histopathology and may offer speed and accuracy in the clinical setting, which would decrease operating costs and waiting time for the patient. In this feasibility study, DSCM showed potential for accurate, rapid, and easy-to-interpret clinical screening of a tumor in whole tissue excisions. Alternative techniques for detecting BCC include Raman spectroscopy ${ }^{6,7}$ and imaging using the contrast of autofluorescence in combination with amino levulinic-induced protoporphyrin IX fluorescence. ${ }^{8}$ Though potentially tumorspecific, these techniques currently lack the spatial resolution to locate small tumors and suffer from autofluorescence contamination; weak signals that require long detection times, sparse sampling and extensive data processing requirements remain challenges in the clinic. More precise tumor location can be obtained with fluorescence polarization microscopy ${ }^{9}$ and by staining cells with toluidine blue. ${ }^{10}$ These confocal techniques can precisely locate small tumors but do not currently combine modes with digital staining. The DSCM reproduced the appearance of H\&E staining, which facilitated interpretation and was well received by the surgeons and pathologists. In some cases, saturation in the gray-scale fluorescence mosaics led to conglomeration of nuclei in images of nodular tumors. This saturation artifact that occurred in the off-line analysis would not be a problem in clinical practice because the laser illumination power will be adjusted for optimum brightness dynamic range to identify cell borders.

One artifact in the DSCM was the occasional presence of purple coloring in the dermis. Acridine orange was occasionally retained in fibrous dermal components. Correlation with the histopathology showed that the fluorescently labeled fibers corresponded to regions of solar elastosis, a condition common in older, sun-damaged skin. To further refine the staining mechanism, lower concentrations of acridine orange will be tested. The use of diluents and rinsing procedures will also be investigated. The reflectance mode introduced speckle noise apparent in [Figs. 1(g)-1(i)]. A future cost-benefit analysis will explore candidates for a second fluorescence dye to replace the reflectance contrast.

Future investigation, including principal component analysis and colorimetry of $\mathrm{H} \& \mathrm{E}$, may maximize contrast and ease of interpretation and trigger the conditioned recognition of clinicians trained for histopathology.

The multimodal mosaics in this study were acquired with a prototype system, and the DSCMs were produced off-line for this preliminary feasibility study. The microscope, built for proof of principal, was slow, requiring 5-15 $\mathrm{min}$. The most time-consuming steps for DSCM were the physical scanning step-and-capture routine and the frame-stitching software run time. Future methods will either acquire each frame in both modes simultaneously or acquire the frames sequentially while the mosaics are acquired simultaneously. The step and capture routine will be nearly twice as fast and offer improved microscopic correlation. The digital stitching of image frames to yield a mosaic, described here, may also be refined for speed. Software and device integration is needed to achieve the optimal speed of this technique, which is likely as small as 3 min. If nontoxic staining or an endogenous label-free optical signature for nuclei is developed, then the technique could be used intraoperatively instead of histopathology in the Mohs procedure to render a rapid diagnosis without freezing or cutting artifacts.

Studies underway will include more samples and evaluate sensitivity and specificity as in Ref. 4 . This technique is currently being adapted for squamous cell carcinoma, which is also routinely screened with H\&E in Mohs surgery. As H\&E histopathology is extremely common, DSCM may also provide a means for rapid surgical pathology at the bedside for excisions of other tissues, such as oral, esophageal, head and neck, breast, liver, and bladder. This must be tested in the context of other tumor types. Though occasional dye retention in non-nuclear material must be addressed, the overall contrast of the confocal reflectance and fluorescence modes mirrored the conventional labeling of tissue by H\&E well. Subsequently, appearance of DSCM mimicked the appearance of $\mathrm{H} \& \mathrm{E}$ pathology. In this mosaicing work [see Fig. 1(i)], the stitching algorithm analyzed the fluorescence mosaic and the corresponding reflectance mosaic was assumed to have the same intertile displacement. Future studies will implement scanning that collects the modes simultaneously, making this assumption correct. This feasibility study showed that the technique presents an attractive alternative to $\mathrm{H} \& \mathrm{E}$ for intraoperative use because it can produce a similar appearance quickly.

\section{Acknowledgments}

Special thanks to Ricardo Toledo-Crow, Charles DiMarzio, Milind Rajadhyaksha, Steven Jacques, and Kevin Phillips for scientific collaboration and critical review of this paper, Jay Eastman and Bill Fox of Lucid Inc. for technical support, histotechnicians Marie Tudisco and Barbara Strippoli for supplying Mohs specimens, Kishwer Nehal and Klaus Busam for guidance in surgical pathology, and Manish Kenkre and Ivanka Kovalyshyn for help with DSCM image preparation.

\section{References}

1. M. Rajadhyaksha, G. Menaker, T. J. Flotte, P. J. Dwyer, and S Gonzalez, "Rapid confocal examination of non-melanoma cancers in skin excisions to potentially guide Mohs micrographic surgery," $J$. Invest. Dermatol. 117, 1137-1143 (2001).

2. D. S. Gareau, Y. G. Patel, Y. Li, I. Aranda, A. C. Halpern, K. S. Nehal, and M. Rajadhyaksha, "Confocal mosaicing microscopy in skin excisions: a demonstration of rapid surgical pathology," J. Microsc. 233(1), 149-159 (2009).

3. D. S. Gareau, Y. Li, B. Huang, Z. Eastman, K. S. Nehal, and M. Rajadhyaksha, "Confocal mosaicing microscopy in Mohs skin excisions: feasibility of rapid surgical pathology," J. Biomed. Opt. 13, 054001 (2008)

4. J. K. Karen, D. S. Gareau, S. W. Dusza, M. Tudisco, M. Rajadhyaksha, and K. S. Nehal, "Detection of basal cell carcinomas in Mohs excisions with fluorescence confocal mosaicing microscopy," $\mathrm{Br} . J$. Dermatol. 160(6), 1242-1250 (2009). 
5. N. A. Swanson, "Mohs surgery: technique, indications, applications, and the future," Arch. Dermatol. 119(9), 761-773 (1983).

6. A. Nijssen, K. Maquelin, L. F. Santos, P. J. Caspers, T. C. Bakker Schut, J. C. den Hollander, M. H. A. Neumann, and G. J. Pupples, "Discriminating basal cell carcinoma from preilisional skin using high wave-number Raman spectroscopy," J. Biomed. Opt. 12(3), 034004 (2007).

7. C. A. Lieber, S. K. Majumder, D. Billheimer, D. L. Ellis, and A. Mahadevan-Jansen, "Raman microscopy for skin cancer detection," J. Biomed. Opt. 13(2), 024013 (2008).

8. M. B. Ericson, J. Uhre, C. Strandeberg, B. Stenquist, O. Larko, A. M.
Wennberg, and A. Rossen, "Bispectral fluorescence imaging combined texture analysis and linear discrimination correlation with histopathologic extent of basal cell carcinoma," J. Biomed. Opt. 10, 034009 (2005).

9. A. N. Yaroslavski, E. V. Salomatina, V. Neel, R. Anderson, and T. Flotte, "Fluorescence polarization of tetracycline derivatives as a technique for mapping nonmelanoma skin cancers," J. Biomed. Opt. 12(1), 014005 (2007).

10. M. Y. Al-Arashi, E. Salomatina, and A. N. Yaroslavsky, "Multimodal confocal microscopy for diagnosing nonmelanoma skin cancers," $\mathrm{La}$ sers Surg. Med. 39, 696-705 (2007). 